\title{
BLOOD VESSEL IDENTIFICATION AND SEGMENTATION FROM RETINAL IMAGES FOR DIABETIC RETINOPATHY
}

\author{
Dr. S. V. Viraktamath \\ Department of E\&CE, \\ SDM College of Engineering \& \\ Technology \\ Dharwad, Karnataka State, India
}

\author{
Deepak Hiremath \\ Department of E\&CE, \\ SDM College of Engineering \& \\ Technology \\ Dharwad, Karnataka State, India
}

\author{
Kshama Tallur \\ Department of E\&CE, \\ SDM College of Engineering \& \\ Technology \\ Dharwad, Karnataka State, India
}

\begin{abstract}
Many diseases can be detected by examining the blood vessels of the retina, some of them are Hypertension, Glaucoma, Cardiovascular diseases, Diabetic retinopathy etc. In the early stages of above diseases blood vessels show early deformities or abnormalities. In this paper the extraction, identification and segmentation of blood vessels from retinal images have been presented, these results are compared with the database of the healthy blood vessels of the retina to detect the infected blood vessels.
\end{abstract}

Keywords—Blood vessel; retina; database; blindness;

\section{INTRODUCTION}

Blood vessels segmentation or extraction of retinal images is required for analysis and for early diagnosis, treatment planning for several diseases and stroke. According to world Health Organization (WHO) $81 \%$ of the people who are blind are above age of 50 years, the number of people who are blind are 38.5 million in 2020 and it will increase to 115 million in 2050 [1]. Diabetic retinopathy, a complication of diabetes mellitus, affects up to $80 \%$ of diabetics and it has even caused blindness in developed countries like the USA [2]. Arteriosclerosis is the hardening and thickening of the walls of the arteries which contributes to the development of cardiovascular diseases; it is the one of the main reasons of death for most people over the age group of 45 years. It has an overall prevalence of circa $30 \%$ and hypertension or high blood pressure, which are the main factor for myocardial infarction, stroke, ischemia, and congestive heart failure. According to recent studies, the overall prevalence of hypertension is almost to the $25 \%$ of population.

The retinal membrane may be a distinctive site wherever the blood vessels will be directly pictured non-invasively and in vivo. Nowadays, digital ophthalmoscopes are able to take quite clear pictures of the membrane, with the possibility of storing them in a digital format and offering the opportunity for automated image processing and analysis. Although this idea has attracted the attention of many research teams, the problem is still not completely solved.
The retina is the light-sensitive layer which is present in eye and it is the most important anatomical part of the eye in the context of this work. The retina is a multi-layered tissue consists of different cells for light-energy conversion, the pre-processing of visual information and transmission of the signal to neural part. The photoreceptive layer which is found furthest from the pupil, next to the choroid and pigment epithelium. The double blood supply is provided to the retina from the top and the bottom of the layer the portion which comes through the tissue layer brings sixty fifth of the blood offers and the part which is coming from the top of the retina brings $35 \%$.

\section{LITERATURE SURVEY}

Dr. Pradeep et.al. [3] Presents a work on diabetic retinopathy and its causes. The, explanation and restrictions of retina databases which are used to test the performance of these detection algorithms are presented and the fundus image is filtered then in controlled manner the image is segmented.

Features of the retinal image are extracted using Grey Level Co-occurrence Matrix (GLCM). The databases used are MESSIDOR and DIARETDB0. For MESSIDOR data set the sensitivity is $100 \%$, specificity is $83 \%$ and accuracy is $95 \%$.

The sensitivity is $90 \%$ specificity is $100 \%$ and accuracy is 93\% for DIARETDB0 data set. A new method proposed by M. PonniBala et.al. [4] for classification of blood vessel by using Extreme Learning Machine (ELM) over Support Vector Machine (SVM). The 2D matched filter is used for blood vessel segmentation after pre-processing. DIARETDB0 and DRIVE are the databases used in the work. For DIARETDB0 data set the sensitivity is $96.6 \%$, specificity is $100 \%$ and accuracy is $97.5 \%$. And for DRIVE data set the sensitivity is $100 \%$, specificity is $94.11 \%$ and accuracy is $95 \%$.A method of segmentation by Toufique Soomro et.al. [5] was proposed aiming at improvising the performance of segmenting small vessels. This algorithm includes morphological and filtering method to remove the background noise and uneven illumination. DRIVE and 


\section{International Journal of Engineering Applied Sciences and Technology, 2020 \\ Vol. 5, Issue 6, ISSN No. 2455-2143, Pages 170-175 \\ Published Online October 2020 in IJEAST (http://www.ijeast.com)}

STARE are the databases used in this algorithm. For DRIVE dataset the sensitivity is $74.65 \%$, specificity is $74.65 \%$ and accuracy is $95.15 \%$. And for STARE dataset the sensitivity is $74.98 \%$, specificity is $95.96 \%$ and accuracy is $95.05 \%$.

\section{METHODOLOGY}

Some of the main operations in the process of retinal blood vessels identification and segmentation are as shown in Fig.2. Pre-processing is helps to remove the disturbances of the acquired input retinal images. The retina image captured from the fundus camera will be in the form of RGB image as shown in Fig. 3a.

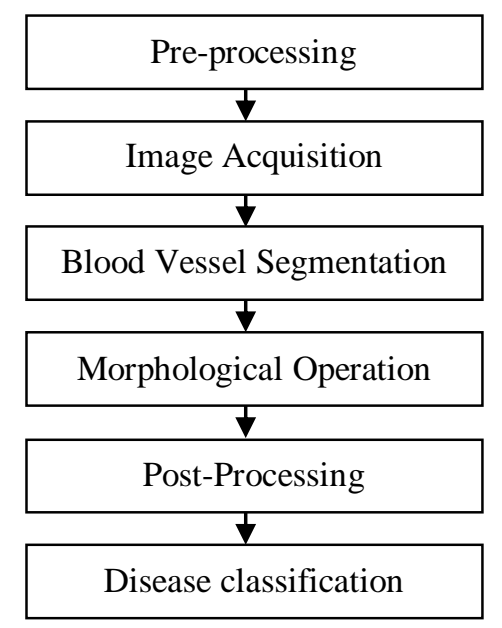

Fig.2. Flow chart of feature extraction

The green channel image consists of more information since there is a higher contrast between the vessel pixels and the non-vessel pixels which is shown in Fig. 3b. ContrastLimited Adaptive Histogram Equalization (CLAHE) is performed. The necessity of filtering the histogram equalized image is to suppress the background pixels. Here a $3 \times 3$ median filter is used to remove the poor illuminated pixel as shown in the Fig. 3c.

Since color fundus images often show important lighting variations, poor contrast and noise, a pre-processing step is needed. The following are the steps involved in this stage : a) Vessel central light reflex removal, b) Background homogenization, and c) Vessel enhancement.

The green layer of the image was isolated, and morphological opening was implemented on it and the resultant image is labeled as $\mathrm{I} \gamma$
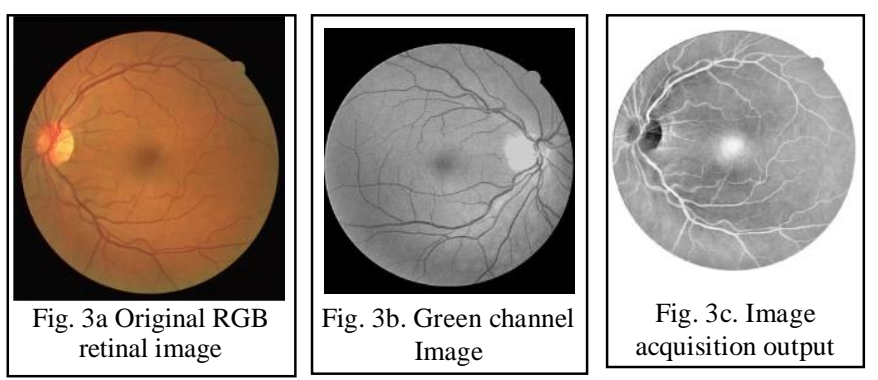
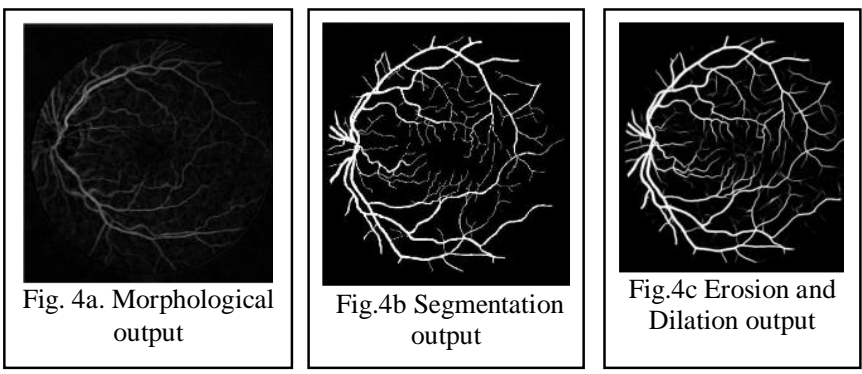

To obtain the shade corrected image of retina which is shown in Fig.4, the background image is removed from the image after the morphological opening as shown in equation (1).

$$
D(x, y)=\mathrm{I} \gamma(\mathrm{x}, \mathrm{y})-\mathrm{Ib}(\mathrm{x}, \mathrm{y})--(1)
$$

Then the image was linearly transformed to cover all possible ranges of gray- levels $[0,255]$. This new image which is obtained is called $\mathrm{I}_{\mathrm{SC}}$. The transform is indicated in below the equation 2 .

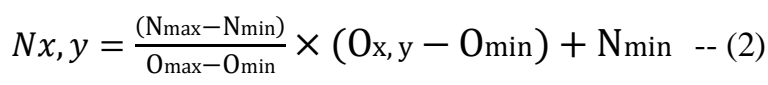

where, Nmax, Nmin, Omax and Omin which are the desired maximum and minimum gray level values of the new, and old histograms respectively; and Ox,y is the value of the pixel which has to be changed.

Finally, to reduce the influence of intensity variations along the image, a homogenized image $\mathrm{IH}$ is obtained displacing toward the middle of the histogram the pixel intensities of the images. This is accomplished by applying the transformation function which is replicated in equation 3 .

$$
\text { goutput }=\left\{\begin{array}{c}
0, \text { if } g<0 \\
255, \text { if } g>255 \quad-\text { (3) } \\
g, \text { otherwise }
\end{array}\right.
$$

Where,

$$
g=g \operatorname{Input}+128-g \operatorname{InputMax}--(4)
$$

Where gInput and gOutput are the gray-level values of the input and output images. gInputMax indicates the mode of the pixel-value intensities in $\mathrm{I}_{\mathrm{SC}}$.

Vessel Enhancement is performed by estimating the complement image of $\mathrm{IcH}$, and then applying a morphological Top-Hat transformation using as a structuring element a disc of eight pixels in radius. The vessel enhanced image is obtained, and IV E can be defined as follows.

$$
\mathrm{I}_{\mathrm{VE}}=\mathrm{I}_{\mathrm{H}}^{\mathrm{c}}-\gamma\left(\mathrm{I}_{\mathrm{H}}^{\mathrm{c}}\right) \quad--(5)
$$

Where, $\gamma$ is the morphological opening operation.

Retinal Vessel Segmentation- After pre-processing, the second matched filter is employed for blood vessel 


\section{International Journal of Engineering Applied Sciences and Technology, 2020 \\ Vol. 5, Issue 6, ISSN No. 2455-2143, Pages 170-175 \\ Published Online October 2020 in IJEAST (http://www.ijeast.com)}

segmentation. The use of filtering the image is to enhance the blood vessels. Blood vessels usually have poor local contrast. The two-dimensional matched filter kernel is designed to enhance the blood vessels to convolve with the original image. The gray-level profile of the cross section of a blood vessel can be approximated by a Gaussian shaped curve. A prototype matched filter kernel is expressed as shown in equation 6 .

$$
f(x, y)=-\exp \left(\frac{-x^{2}}{2 \sigma^{2}}\right), \text { for }|y| \leq \frac{L}{2}--(6)
$$

Where, $\mathrm{L}$ is the length of the segment for which the vessel is assumed to have a fixed direction, $\sigma$ defines the spread of the intensity profile. The parameter $\mathrm{L}$ is chosen to be equal to 30 pixels and $\sigma$ is 8 . The direction of the vessel is assumed to be aligned along the y-axis. Since a vessel may be oriented at any angles, the kernel needs to be rotated for all possible angles. A set of 12 different kernels is applied by convolving to a retinal image. The matched filter image is processed by a proper thresholding scheme which can be used to differentiate between enhanced vessel segments and the background. The thresholding method developed by Chanwimaluang is used for retinal vessel thresholding. A smoothed co-occurrence matrix is developed to increase the entropy and to reduce the peak in the co-occurrence. By considering the vertically lower and horizontally right transitions, the co-occurrence matrix is given in equation (7).

$$
t_{i j}=\sum_{l=1}^{P} \sum_{k=1}^{Q} \delta(l, k)--(7)
$$

Where,

$$
\begin{aligned}
& (l, k)=1 \text { if }\left\{\begin{array}{c}
\mathrm{f}(\mathrm{l}, \mathrm{k})=\mathrm{i} \text { and } \mathrm{f}(\mathrm{l}, \mathrm{k}+1)=\mathrm{j} \\
\text { or } \\
\mathrm{f}(\mathrm{l}, \mathrm{k})=\mathrm{i} \text { and } \mathrm{f}(\mathrm{l}+1, \mathrm{k})=\mathrm{j}
\end{array}\right. \\
& (l, k)=0 \quad \text { otherwise-- }(9)
\end{aligned}
$$

$p i j$, the probability of co-occurrence of gray levels $i$ and $j$ is given by equation 10 .

$$
p i j=\frac{\mathrm{tij}}{\sum i \sum j t_{i j}} \quad--(10)
$$

\section{DATABASES USED IN RETINAL IMAGE PROCESSING}

Medical image databases with an appropriate Ground Truth (GT) about the image contents enable the development and proper evaluation of automatic image analysis methods. In this section, databases containing GT for the blood vessels and databases containing GT for the estimation of Arteriolar-to-Venular Ratio (AVR) are reviewed. Five publicly available databases were identified, which have the GT for the blood vessels: ARIADB, CHASEDB1, DRIVE, HRF and STARE. The data used for validation of the proposed approach is obtained from the publicly available DRIVE database. The screening population consisted of four hundred diabetic subjects between 25-90 years archaic.
Some haphazardly selected images about forty among which 33 do not show any sign of diabetic retinopathy and 7 show signs of mild early diabetic retinopathy. Every image has been JPEG compressed. The DRIVE database consists of 40 color fundus images grouped into two sets: test and training each contain 20 images with a resolution of $584 \times 565$ pixels. Among 40 images, 33 are images with no symptoms of pathology and 7 are images which show signs of diabetic retinopathy. Three images are in the training set and four are present in the test set out of total seven images. For clinical diagnosis all 40 images are used. This database has been extensively used for validation of retinal blood vessel segmentation techniques. Hence, for comparison purpose the work on the DRIVE database has been tested and validated.

After segmentation of the fundus retina image a binary image is created which consists only the blood vessels of retina. The performance of this process of segmentation is understood by calculating some parameters like True Positive (TP), True Negative (TN), False Positive (FP) and False Negative (FN).

Specificity: Specificity is the percentage of non-vessel pixels that are correctly classified as non-vessel pixels.

$$
\text { Specificity }=\mathrm{TN} /(\mathrm{TN}+\mathrm{FP}) \text {-- (11) }
$$

Sensitivity: Sensitivity is the percentage of the actual vessel pixels that are detected.

$$
\text { Specificity }=\mathrm{TP} /(\mathrm{FN}+\mathrm{TP})--(12)
$$

Accuracy: The ratio between the total numbers of correctly classified instances and the test size is called as accuracy.

$$
\text { Accuracy }=(\mathrm{TP}+\mathrm{TN}) /(\mathrm{TP}+\mathrm{TN}+\mathrm{FP}+\mathrm{FN})--(13)
$$

Where,

$\mathrm{TN}=$ truly classified pixels that are non-vessel pixels.

$\mathrm{TP}=$ truly classified pixels that are vessel pixels.

$\mathrm{FN}=$ No. of pixels that have falsely classified as non-vessel pixels.

FP $=$ No. of pixels that have falsely classified as vessel pixels.

Performance parameter can be understood by using Fig.5(a) and Fig.5 (b). if the white pixels showing in Fig.5(a) is same as in the Fig.5(b) then it is classified as TP if not then TN. Similarly, if the black pixels showing in Fig.5 (a) is same as in the Fig.5 (b) then it is classified as FP if not then FN. This can be done by comparing every pixel of the Fig.5(a) to the all pixels of Fig 5(b).
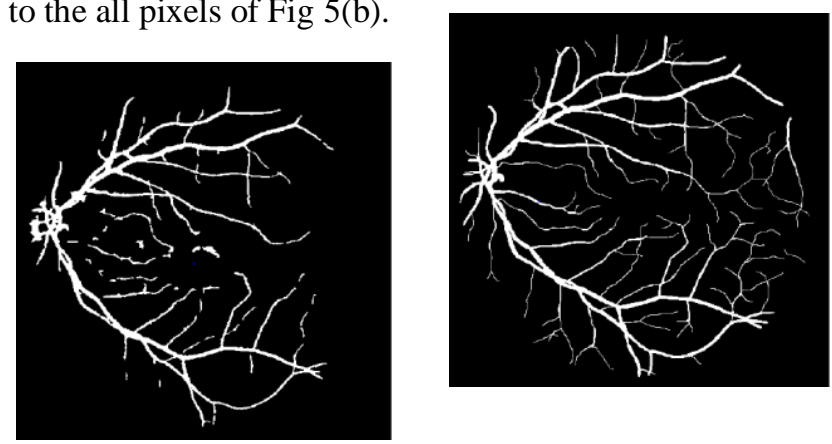


\section{International Journal of Engineering Applied Sciences and Technology, 2020 Vol. 5, Issue 6, ISSN No. 2455-2143, Pages 170-175 \\ Published Online October 2020 in IJEAST (http://www.ijeast.com)}

Fig. 5a. Generated binary image Fig. 5b. Manually segmented image

The algorithms are tested most commonly on the publicly available DRIVE database. This database consists of 40 images, with 20 images in test set and 20 images in training set with the resolution of 584 x 565 for each image.

\section{RESULTS AND DISCUSSIONS}

Test on DRIVE Database- This algorithm is tested on DRIVE database which is publicly available for academic purpose. The variation in the extracted output blood vessels as it converts the RGB channel into different channels like Red, Green or Blue is shown in Fig.6a, Fig.6b and Fig.6c In the Red channel output image, it is seen that the low frequency information in the images are not clearly classified hence performance is reduced. The Green channel output image shows that all the blood vessels are clearly identified, and this channel is generally used as default channel for any feature extraction from the 2D images. Similarly, blue channel will reduce the performance due to the fewer light elements in this channel hence it is difficult to identify the blood vessels from this channel.

As described above, after morphological operation the gray threshold level of these mi binary image (morphological output) is calculated. This level can be varied to get good response from the images. Thus, by subtracting a small value from the threshold level will result in accommodating more blood vessels in the output binary image which is shown in Fig.7a, Fig.7b and Fig.7c. Comparison with Other Algorithms- The blood vessels extraction performance results for drive data base is shown in table1.
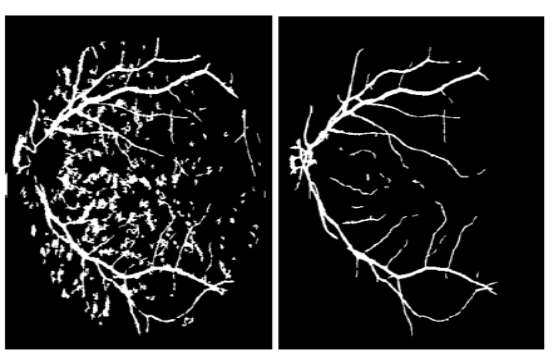

Fig.6a Red channel Fig.6b Green channel

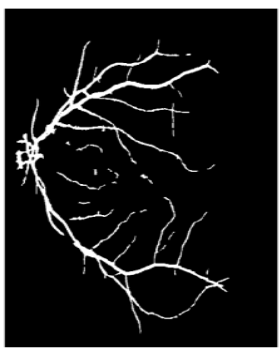

Fig.7a Level

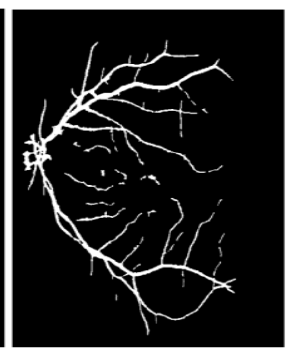

Fig.7b Level-0.01

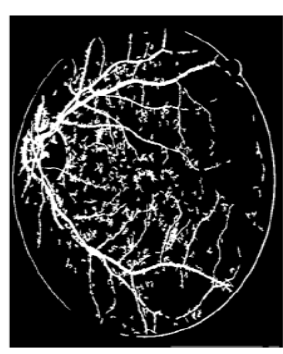

Fig.6cBlue channel

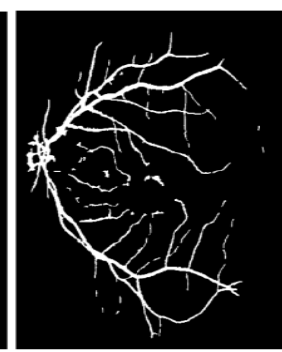

Fig.7c Level-0.02
The performance of the proposed algorithm is compared with the other algorithm in terms of Accuracy, Sensitivity and Specificity. Table 2 shows the comparison of the proposed algorithm with the others.

Table.1 Blood Vessels Extraction Performance Results for DRIVE database.

\begin{tabular}{|c|c|c|c|}
\hline Image name & Accuracy & Sensitivity & Specificity \\
\hline 21_training.tif & 96.7299 & 71.2588 & 98.7871 \\
\hline 22_training.tif & 95.7683 & 66.6678 & 98.6583 \\
\hline 23_training.tif & 81.0944 & 85.7386 & 80.7671 \\
\hline 24_training.tif & 9406575 & 61.1028 & 99.0546 \\
\hline 25_training.tif & 95.1967 & 60.8734 & 98.8407 \\
\hline 26_training.tif & 94.64 & 75.5159 & 96.3838 \\
\hline 27_training.tif & 95.8237 & 67.6057 & 98.5503 \\
\hline 28_training.tif & 95.4937 & 68.2729 & 98.4402 \\
\hline 29_training.tif & 95.8349 & 70.3398 & 98.1761 \\
\hline 30_training.tif & 94.9627 & 74.1539 & 96.734 \\
\hline 31_training.tif & 93.3286 & 77.3143 & 94.3563 \\
\hline 32_training.tif & 96.2862 & 73.0359 & 98.357 \\
\hline 33_training.tif & 96.1077 & 76.7893 & 97.8076 \\
\hline 34_training.tif & 86.9184 & 80.311 & 87.6351 \\
\hline 35_training.tif & 95.918 & 71.512 & 98.2353 \\
\hline 36_training.tif & 94.4269 & 69.9671 & 97.4116 \\
\hline 37_training.tif & 95.0627 & 66.4263 & 97.8055 \\
\hline 38_training.tif & 95.1424 & 76.2651 & 96.9255 \\
\hline 39_training.tif & 95.1803 & 76.5521 & 96.9312 \\
\hline 40_training.tif & 95.9028 & 80.4248 & 97.1718 \\
\hline Average & 94.22 & 72.5 & 96.35 \\
\hline
\end{tabular}

Table.2 Comparison of proposed method with other algorithms for DRIVE Database

\begin{tabular}{|c|c|c|c|}
\hline Author & Accuracy & Sensitivity & Specificity \\
\hline $\begin{array}{c}\text { Palomera } \\
\text { et.al[11] }\end{array}$ & 92.20 & 66.00 & 96.10 \\
\hline Khan et.al[12] & 95.80 & 73.00 & 97.93 \\
\hline Jiang[13] & 92.22 & 64.78 & 96.25 \\
\hline Proposed method & 94.22 & 72.50 & 96.35 \\
\hline
\end{tabular}

Figure 8a, Fig 8b, fig 8c shows the Accuracy, Sensitivity and Specificity for different channels. Figure 8d, fig 8e, fig 8f shows the Accuracy, Sensitivity and Specificity for different threshold levels.

$$
\rightarrow \text { RED } \rightarrow \text { GREEN } \leadsto \text { BLUE }
$$

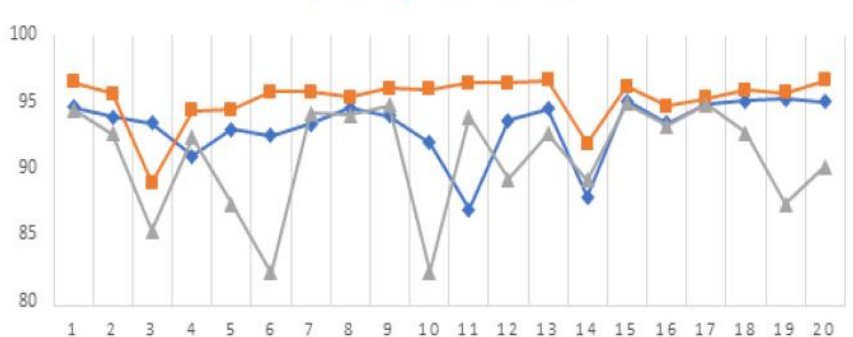

Fig.8a. Accuracy for different channels. 


\section{International Journal of Engineering Applied Sciences and Technology, 2020 \\ Vol. 5, Issue 6, ISSN No. 2455-2143, Pages 170-175 \\ Published Online October 2020 in IJEAST (http://www.ijeast.com)}

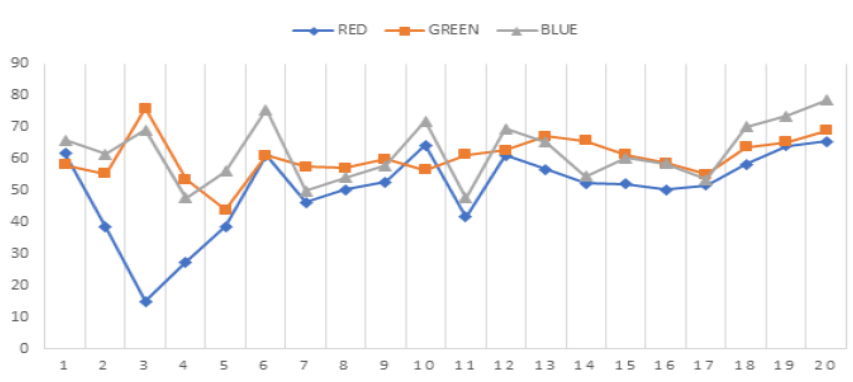

Fig.8b. Sensitivity for different channels.

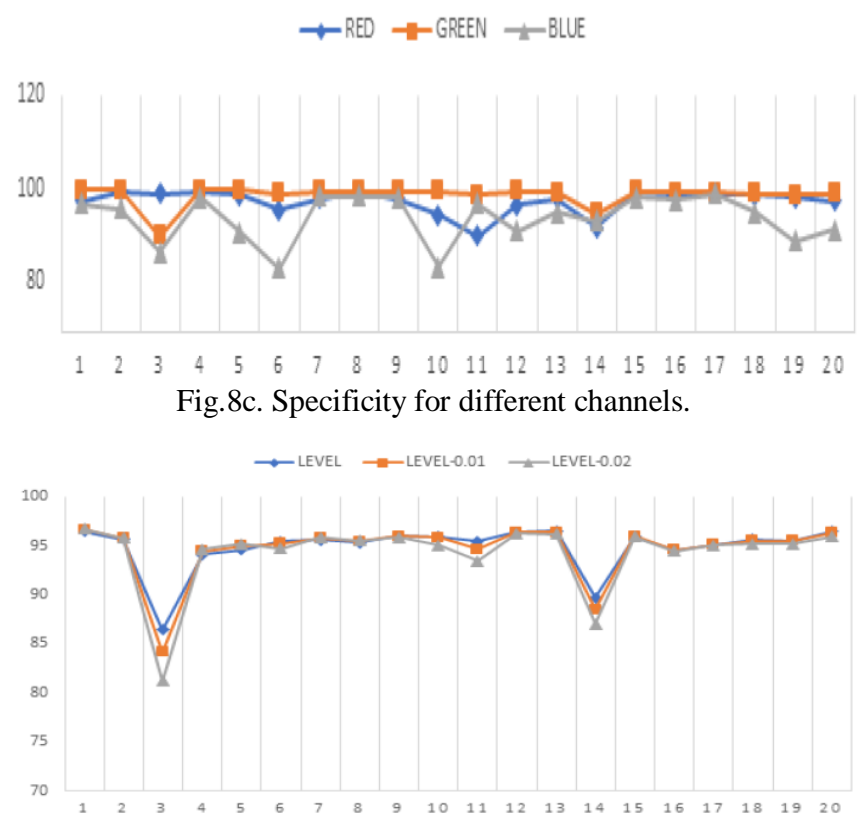

Fig.8d. Accuracy for different threshold levels.

Machine Learning (ML) based classification KNN classifier may be used to find diabetic retinopathy (DR). It is one of the simplest but widely using machine learning algorithms. Linear Comparison Classifier in which linear pixel to pixel comparison is used to understand percentage of similarities between the given input image and the available set of images. In this method the given input fundus image is segmented as described in Fig.9 and it is compared pixel by pixel with every image in database. This database consists of binary images of retina. Due to lack of segmented images available in database we were unable to classify the level of DR disease, but we proposed a linear comparison method to identify whether the fundus image is under DR or not. The only condition in linear comparison classifier is that the given input fundus image should have a similar binary image in the database.

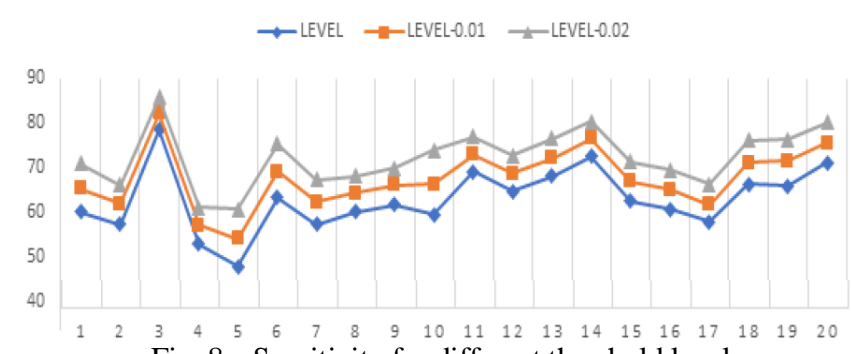

Fig. 8e. Sensitivity for different threshold levels.

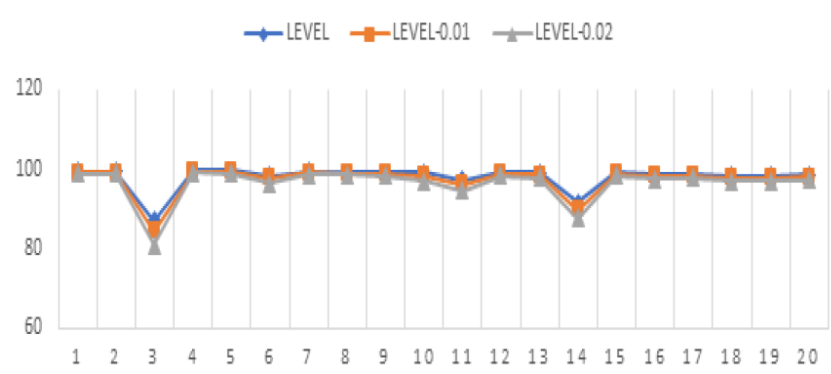

Fig. 8f. Specificity for different threshold levels.

If the similar binary does not available in the database, then it shown incorrect results. The Fig. 10a and Fig. 10b shows the screenshots of the linear classifier to classify the given retina image as either healthy or DR affected.

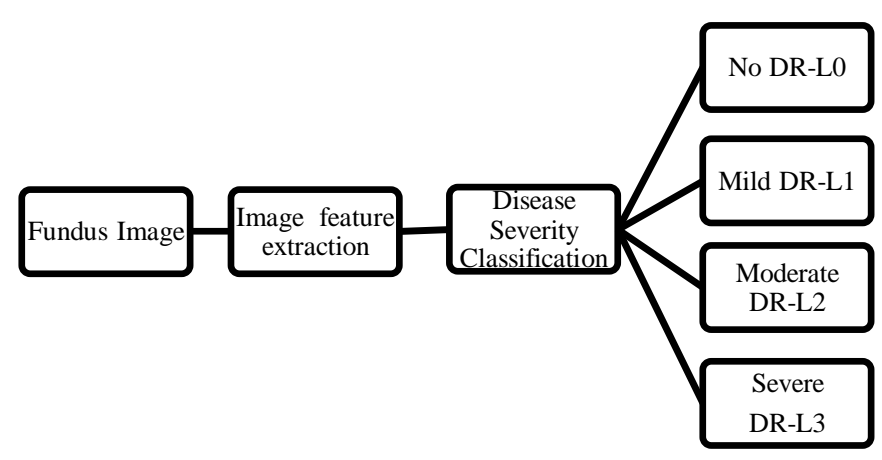

Fig. 9 The processes of DR Classification system 


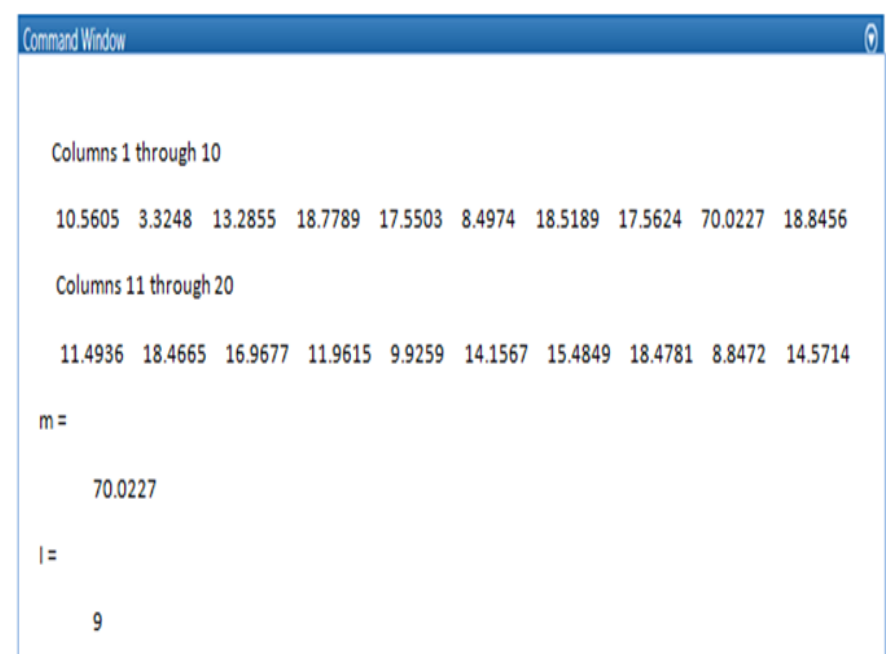

Fig. 10a. Example to classify fundus image as DR.

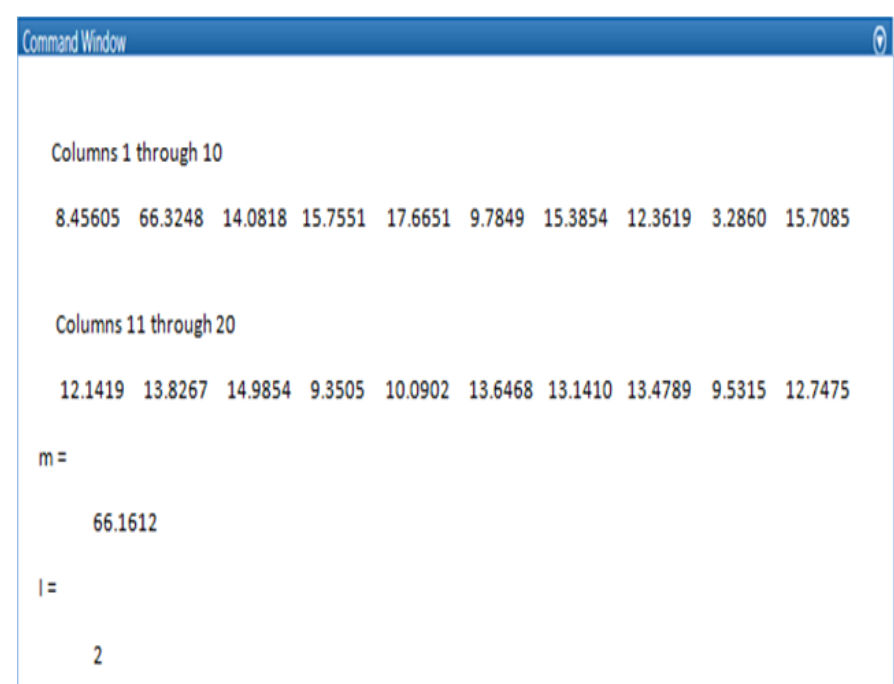

Fig. 10b. Example to classify fundus image as healthy.

\section{CONCLUSION}

This paper proposed one modified algorithm for blood vessels identification/extraction and segmentation by converting a colored fundus image into green channel image. The image is represented into inverse histogram for better results. To filter the unwanted parts of the image, those images are passed through the morphological filters. A single local threshold is used to create a binary image in the last part of the algorithm. A publicly available DRIVE Database is used to test this method of vessels extraction and calculated the Accuracy, Sensitivity and Specificity as $94.22 \%, 72.50 \%$ and $96.35 \%$ respectively.

\section{ACKNOWLEDGMENT}

The authors wish to thank K-Tech Innovation Hub, New Age Innovation Network Center, District Innovation Center,
Dharwad Region, S D M College of Engineering and Technology, Dhavalagiri DHARWAD.

\section{REFERENCE}

[1]. Ramakrishnan Sundaram, Ravichandran K S, Premaladha Jayaraman D and Venkatraman B, "Extraction of Blood Vessels in Fundus Images of Retina through Hybrid Segmentation Approach". $\begin{array}{lll}\text { Mathematics } & \text { 2019, } & 7(2),\end{array}$ https://doi.org/10.3390/math7020169.

[2]. Ming-Yee Chiu, Depommer R., Spindler T. Pattern Recognition, 2004, "An embedded real-time Vision system for 24-hour indoor/outdoor car-counting applications".

[3]. Lee, S. 2007, "Intelligent Liquid Crystal Display (iLCD) for Next Generation Television Applications". IEEE Transactions on Consumer electronics 53(4) DOI-10.1109/tce.2007.4429208.

[4]. Song. J, Xi. N, Xu. F, Jia. K, \& Zou. F. (2015). Servomotor modelling and control for safe robots. IEEE International Conference on Robotics (ROBIO). DOI:10.1109/robio.2015.7418938.

[5]. Marshall C, Parker T, \& White T, (n.d.). "Infrared sensor technology". Proceedings of 17th International Conference of the Engineering in Medicine and Biology Society. DOI:10.1109/iembs.1995.579906.

[6]. B. Nath, F. Reynolds, R. Want (2006) "RFID Technology and Applications". IEEE Pervasive computing, 5(1), 2-24.DOI: 10.1109/MPRV.2006.13.

[7]. Badamasi, Y. A, 2014. "The working principle of an Arduino". 11th International Conference on Electronics, Computer and Computation (ICECCO). doi:10.1109/icecco.2014.6997578

[8]. Mr. Basavaraju S R, "Automatic Smart Parking System using Internet of Things (IOT)", International Journal of Scientific and Research Publications, Volume 5, Issue 12, December 2015629 ISS 2250-3153.

[9]. Galkin I, \& Tetervenoks O. 2014. "The study of microcontroller based embedded system for smart lighting applications". 6th European Embedded, Design in Education and Research Conference (EDERC). doi:10.1109/ederc.2014.6924368.

[10]. Md Kamal M, \& Mamat N. 2009. "Controller Design for Servo Motor”. IEEE Symposium on Industrial Electronics \& Applications. doi:10.1109/isiea.2009.5356325.

[11]. Yasa Y, Sahin E, Acar C, Gozutok A, Firat E, \& Mese E. 2013. "Servo Motor Driver Design for High Performance doi:10.1109/epecs.2013.6713062. Applications". 